\title{
Combustibles alternativos en el transporte aéreo nacional
}

| Fecha de recibido: 22 de noviembre del 2019 | Fecha de aprobación: 23 de septiembre del 2020 |

\section{Luisa Mónico}

Doctora en Sistemas Propulsivos

en Medios de Transporte

Universidad de San Buenaventura

Grupo de Investigación Aerotech

Rol de investigador: teórico

https://orcid.org/0000-0002-3597-6332

\Imonico@usbbog.edu.co

\section{Estefanía Rincón}

Ingeniera aeronáutica

Universidad de San Buenaventura

Rol de investigador: teórico

https://orcid.org/0000-0001-8149-0950

凶 esrincon@academia.usbbog.edu.co

\section{Cristian Manuel Manrique Vega}

Ingeniero aeronáutico

Universidad de San Buenaventura

Rol de investigador: teórico

https://orcid.org/0000-0002-1152-9271

\.mmanrique@academia.usbbog.edu.co

* Artículo de reflexión derivado del proyecto de grado "Estado del arte de los combustibles alternativos en el sector transporte en Colombia" desarrollado en el pregrado de Ingeniería Aeronáutica de la Universidad de San Buenaventura, sede Bogotá.

Cómo citar este artículo: Mónico, L., Rincón, E., \& Manrique Vega, C. M. (2020). Combustibles alternativos en el transporte aéreo nacional. Revista Ciencia y Poder Aéreo, 15(2), 68-76. https://doi.org/10.18667/cienciaypoderaereo.643 


\section{Combustibles alternativos en el transporte aéreo nacional}

\section{Alternative Fuels in National Air Transport}

\section{Combustíveis alternativos no transporte aéreo nacional}

Resumen: en la actualidad, la alta demanda de pasajeros en los diferentes aeropuertos del país, el crecimiento, fortalecimiento y nacimiento de compañías aéreas junto a la necesidad de encontrar un combustible sustituto del combustible fósil y que a la vez, sea amigable con el medio ambiente, ha potencializado el estudio, implementación, uso y optimización de los combustibles alternativos en el sector de la aviación. Hoy en día, a pesar de que no se tienen numerosos ni grandes avances en la industria de la aviación, la implementación de nuevas fuentes de energía es un tema que se ha venido evaluando con el paso de los años. Por ello, surge la necesidad de conocer el estado actual del uso de estas nuevas energías, específicamente en el sector aéreo nacional, ya que se ha demostrado que Colombia tiene un gran potencial en la producción de biocombustibles a base de caña de azúcar y palma, los cuales han empezado a usarse para el funcionamiento de motores de combustión interna alternativos (gasolina y diésel). Por otra parte, la revisión bibliográfica permitió afirmar que a pesar de los escasos intentos por demostrar las grandes ventajas que tiene el uso de combustibles alternativos en la aviación nacional, los resultados han sido satisfactorios, lo cual promueve al desarrollo y empleo de una nueva fuente de energía en Colombia.

Palabras clave: aviación; biocombustibles; emisiones contaminantes.

Abstract: Today, the high demand of passengers in the different airports of the country, the growth, consolidation and birth of airlines, along with the need to find a substitute for fossil fuels that is also friendly to the environment, have strengthened the study, implementation, use and optimization of alternative fuels in the aviation sector. Despite the fact that not many or major advances have been achieved in the aviation industry, the implementation of new energy sources has been an issue repeatedly evaluated over the years. Thus the need to address the current state of the use of new energies, specifically in the national air sector, considering that Colombia has shown great potential for the production of biofuels from sugar cane and oil palm crops, which have begun to be used for the operation of alternative internal combustion engines (gasoline and diesel). The bibliographic review presented in this work allows stating that despite the limited attempts to demonstrate the advantages of the use of alternative fuels in national aviation results on this regard have proved satisfactory, which promotes the development and use of these new sources of energy in Colombia.

Keywords: Aviation; biofuels; polluting emissions.

Resumo: Hoje em dia, a alta demanda de passageiros nos diversos aeroportos do país, o crescimento, fortalecimento e nascimento das companhias aéreas e a necessidade de encontrar um combustível substituto para o fóssil que também seja amigável com o meio ambiente tem potencializado o estudo, implantação, uso e otimização de combustíveis alternativos no setor da aviação. Na atualidade, apesar de não ter muitos ou grandes avanços na indústria da aviação, a implantação de novas fontes de energia é um assunto que vem sendo avaliado ao longo dos anos. Por isso, surge a necessidade de conhecer o estado atual do uso dessas novas energias, especificamente no setor aéreo nacional. Graças a que está demonstrado que a Colômbia possui grande potencial na produção de biocombustíveis a partir da cana-de-açúcar e da palma, visto que passaram a ser utilizados para o funcionamento de motores alternativos de combustão interna (a gasolina e a diesel). A revisão bibliográfica permite afirmar que mesmo que as poucas tentativas das grandes vantagens do uso de combustíveis alternativos na aviação nacional, e embora os resultados têm sido satisfatórios, o que favorece o desenvolvimento é o uso desta nova fonte de energia na Colômbia.

Palavras-chave: Aviação; Biocombustíveis; Emissões de poluentes. 


\section{Introducción}

Con el paso de los años, las industrias alrededor del mundo han buscado soluciones para mitigar el impacto negativo de los combustibles convencionales. De acuerdo con las cifras del Banco Mundial, el $80 \%$ de la energía que se consume en la tierra proviene del petróleo, lo que ha llevado a que los gobiernos de distintos países diseñen estrategias para contrarrestar el uso de los combustibles de origen fósil (Portafolio, 2016). Se espera que estas energías alternativas sean más amigables con el medio ambiente, ya que la polución del aire es uno de los problemas más graves de la actualidad y uno de los principales causantes de costos de salubridad, junto con la contaminación hídrica, catástrofes naturales y enfermedades (Mónico-Muñoz, Cabezas-Paredes \& Buitrago-Triana, 2018). En suma, una de las alternativas que mejores resultados ha mostrado con el tiempo es el empleo de biocombustibles, los cuales se destacan por su facilidad de producción, utilización, almacenamiento y potencial para reducir los niveles de partículas $\mathrm{HC}$, $\mathrm{CO}_{\text {y } \mathrm{CO}_{2}}$ (Torregrosa et al., 2013).

En el sector aéreo, los combustibles son uno de los factores fundamentales que afectan el crecimiento de esa industria. Hacen parte de los insumos de mayor costo en la operación de una aerolínea (Martín-Crespo, 2016). Al respecto, los costos del transporte aéreo determinan la capacidad de competitividad de las distintas entidades en este medio a nivel global, por tanto, cualquier variación en los precios del combustible puede afectar la economía del sector (Briceño \& López, 2011). En razón a lo anterior, se han construido e implementado políticas petroleras en los diferentes procesos para igualar costos, con el fin de que el campo industrial aéreo sea más eficiente y productivo en sus actividades.

Por otra parte, en el estudio realizado por la Organización de Aviación Civil Internacional (OACI), en la nota A39-WP/55 (OACI, 2016), se evaluaron los niveles de emisiones producidos por los motores de las aeronaves que afectan la calidad del aire. En la figura 1, se visualizan las tendencias respecto a las emisiones que se producen actualmente. En general, se observa que la aviación durante el 2010 consumió cerca de 142 toneladas métricas de combustible, cifras a partir de las cuales se estima que para el 2040, el tráfico internacional de aviación incrementará 4,2 veces, haciendo que el consumo de combustible aumente entre 2,8 y 3,9 veces, lo cual quiere decir que, los niveles de contaminación serían extremadamente altos.

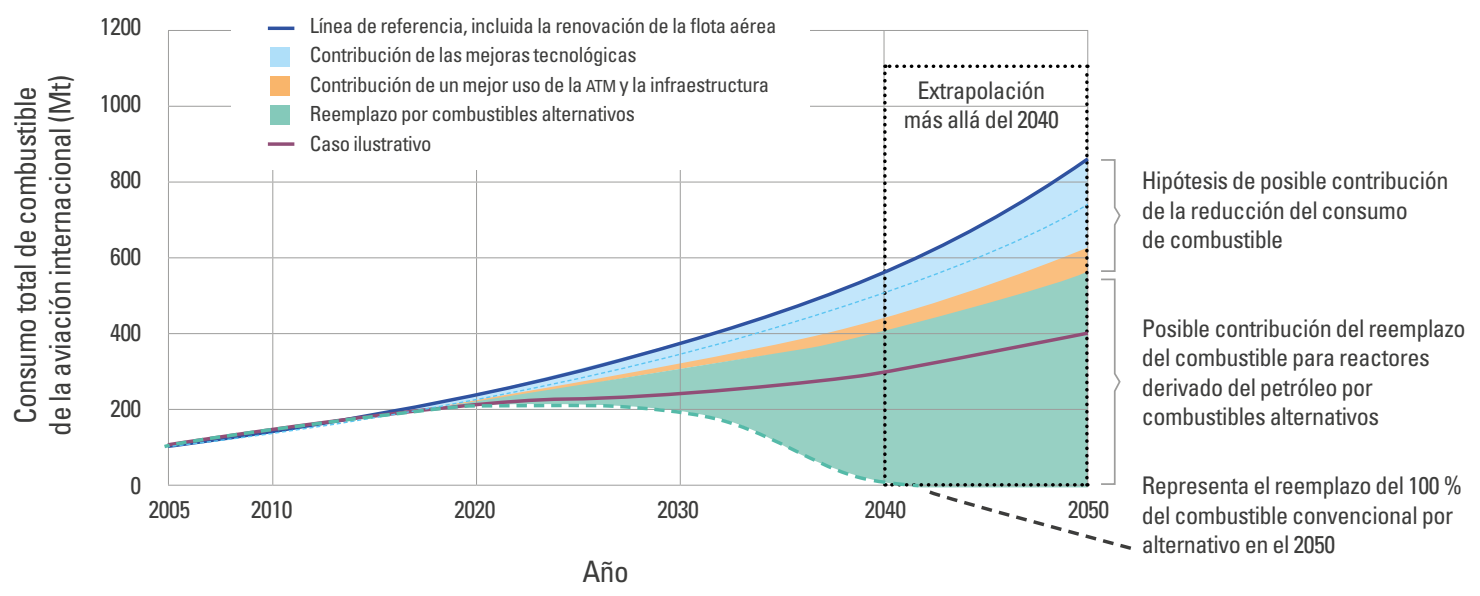

Figura 1. Posible sustitución de combustible fósil por combustibles alternativos en la aviación Fuente: OACI (2016). 
De igual modo, el Grupo Intergubernamental de Expertos en Cambio Climático (IPCC) de las Naciones Unidas ha desarrollado investigaciones en el sector de la aviación, concluyendo que del total de $\mathrm{CO}_{2}$ producido por las diversas industrias, la aviación produce alrededor del $2 \%$ de estas emisiones (Avianca Holdings, 2018; 2019). En el 2018, el $70 \%$ de las emisiones generadas por los motores de aeronaves era dióxido de carbono $\left(\mathrm{CO}_{2}\right)$, seguido por un $29 \%$ de agua $\left(\mathrm{H}_{2} \mathrm{O}\right)$ y un $1 \%$ de óxidos de nitrógeno $\left(\mathrm{NO}_{\mathrm{x}}\right)$, monóxido de carbono (co) y óxidos de azufre ( $\mathrm{SO}_{\mathrm{x}}$ ).

Por lo anterior, se han venido desarrollando y optimizando los combustibles alternativos, entre los que sobresalen los biocombustibles (biodiésel, bioetanol, biomasa, entre otros), y combustibles sintéticos. Los biocombustibles son obtenidos de aceites vegetales o grasas animales por el proceso de transesterificación, se muestra como un excelente sustituto de los combustibles derivados del petróleo debido a su fácil producción, utilización, almacenaje y significativa reducción de emisiones contaminantes, en especial, de $\mathrm{CO}_{2}$ (hasta un $78 \%$ ). Esto se debe al ciclo cerrado del carbono, en el que, al quemarse el biodiesel, el $\mathrm{CO}_{2}$ liberado a la atmósfera es absorbido por las plantas durante el proceso de fotosíntesis para su crecimiento, plantas que después son procesadas para obtener nuevamente el combustible (Mónico-Muñoz, 2013).

La OACI publicó una nota de estudio en la que se muestra la capacidad de producción de combustibles alternativos. En esta se muestra que en el mundo, a diario se consume el equivalente del combustible de origen fósil que la tierra necesitaría acumular durante mil años empleando energía solar. Teniendo en cuenta las cifras de producción anual, se estima que la industria de los biocombustibles no sería capaz de satisfacer la demanda mundial de energía, ni siquiera para el sector de la aviación. En tal sentido, el biocombustible que más se ha considerado es el de tercera generación, es decir, aquellos que son menos dependientes de los cultivos de alimentos, en el cual el problema principal sigue siendo la escases de tierras para su producción (OACI, 2017).
Por ejemplo, para satisfacer solamente la demanda del sector aéreo mundial, se necesitarían 2,7 millones de $\mathrm{km}^{2}$ de plantaciones de jatropha, lo cual equivale a la tercera parte del territorio de Australia. Las plantaciones de camelina se deberían ampliar 2 millones de $\mathrm{km}^{2}$, y para las algas se requerirían 65 mil km² una extensión equivalente a todo el terreno de Irlanda (OACI, 2017).

\section{Aviación internacional}

A la fecha se han tenido experiencias con combustibles alternativos, como el caso de Air France quien realizó con éxito un vuelo de Toulouse a París en un Airbus A321, con una mezcla del $50 \%$ de combustible de aviación convencional y $50 \%$ de biocombustible en ambos motores, dando como resultado, una reducción de 54 g pasajero/kilómetro (Fedebiocombustibles, 2011). Por su parte, Airbus en unión con Virgin Australia, estudiaron una nueva forma de producir combustibles para la aviación empleando Eucaliptos Mallee, cultivados en la región del cinturón de trigo de Australia Occidental y tratados por medio del proceso de pirolisis para producir el combustible alternativo (Fedebiocombustibles, 2012a).

En esa línea, Boeing, Etihad Airways, Honeywell UOP y el SRBC, se dedican al desarrollo y comercialización de biocombustibles de aviación sostenibles, emplean plantas del desierto regadas con agua de mar, las cuales producen biocombustible de forma más eficiente que otras fuentes y reducen las emisiones de carbono entre un $50 \%$ y un $80 \%$ con respecto a los carburantes fósiles a lo largo de su ciclo de vida ("Boeing estudia", s. f.). Así mismo, Boeing ha trabajado en equipo con la empresa South African Airways, para desarrollar biocombustibles a base de una planta de tabaco ("Boeing se asocia", s. f.). En China, la Corporación Petroquímica de China (Sinopec) recibió la licencia para dar un uso comercial del biocombustible para la aviación, el cual ha ganado popularidad en todo el mundo. Se prevé que para el 2020 , el $30 \%$ 
del combustible para la aviación sea biocombustible ("China permite", s. f.). En ese marco, los tres grandes constructores de aviación, Airbus, Boeing y Embraer, en el 2012 firmaron un acuerdo de intensión y colaboración para trabajar conjuntamente en el desarrollo de biocombustibles de aviación, en espera de que para el 2020 se utilice un $4 \%$ de biocombustible en los aviones (Martín-Crespo, 2016).

También en España, Iberia y Repsol, se realizó un vuelo en un A320 entre Madrid y Barcelona en el cual emplearon $75 \%$ de queroseno tradicional y $25 \%$ de biocombustible a base de carmelina, una planta oleaginosa no comestible. En Estados Unidos, el primer vuelo comercial en utilizar biocombustible fue un avión B737 de Continental Airlines para desarrollar un vuelo de prueba de dos horas desde Houston (Beta Analytic, s. f.). Según reportes, se estima que se logró una reducción en las emisiones de $\mathrm{CO}_{2}$ de $1.500 \mathrm{~kg}$ (Repsol, s. f.). En cuanto a normativas se ha desarrollado el método ASTM, el cual se emplea para el análisis de biocombustibles de aviación y sirve como herramienta de verificación para comprobar la precisión de la cantidad de combustible alternativo en la mezcla (Beta Analytic, s. f.). En el 2017 se realizó el primer vuelo internacional desde Beijing hasta Chicago, ocasión en que la aerolínea Hainan Airlines empleó biocombustible a base de residuos de aceite de cocina (Mucino, 2017).

Teniendo en cuenta lo anterior, los ensayos desarrollados hasta el momento posicionan a los biocombustibles como buenos sustitutos de los combustibles fósiles de aviación. Una de las ventajas del uso de este tipo de combustibles en aeronaves, es que no se requiere una mayor adaptación de los motores ni en su respectiva red de distribución (Guzmán-Galeano, 2013). La International Air Transport Association (IATA), ha establecido que a corto plazo se debería estar usando un $6 \%$ de biojet, es decir, lo equivalente a $8 \mathrm{mil}$ millones de litros de biocombustible para aviones (Fedebiocombustibles, 2012b).

En cuanto a América Latina, México tiene como propósito reducir el bióxido de carbono que genera el transporte aéreo, para lo cual aumentará la producción de biocombustibles en un 15 \% para el 2020 y en un $50 \%$ para el 2040 ("México apuesta", 2015). Argentina, firmó un convenio para el desarrollo de biocombustible aeronáutico entre la Subsecretaría de Transporte Aerocomercial, la Administración Nacional de Aviación Civil, aerolíneas argentinas, YPF, Instituto Nacional de Tecnología Industrial (INTI), Instituto Nacional de Tecnología Agropecuaria (INTA), Secretaría de Ambiente y Desarrollo Sustentable de la Nación y la Cámara Argentina de Biocombustibles, con el proyecto de potencializar el uso y desarrollo de combustibles aeronáuticos (Fedebiocombustibles, 2013).

Por otro lado, el empleo de combustibles sintéticos de queroseno parafínico se ha presentado como otra muy buena opción. La mezcla se produce con residuos de la cosecha del maíz, el azúcar o forestales, que según la Federal Aviation Administration (FAA) podría reducir los niveles de emisiones contaminantes hasta un $85 \%$ (Ferrer, 2020). Según la coordinadora técnica del Clúster Bioturbosina, se han hecho más de 5.000 vuelos con biocombustible alrededor del mundo (Mucino, 2017), lo que contribuye día a día al desarrollo de esta gran alternativa para el cuidado del medio ambiente. El sector de la aviación se ha propuesto para el 2050 reducir en un $50 \%$ las emisiones de $\mathrm{CO}_{2}$ a través del empleo de combustibles de segunda generación (algas, camelina y jatrofa) (Rodríguez, 2016).

\section{Aviación nacional}

En Colombia, el sector aéreo en los últimos años ha venido creciendo en oferta y demanda de pasajeros y transporte de carga. Además, las aeronaves que operan actualmente las diferentes aerolíneas nacionales utilizan combustible de tipo AVGAS y JET-A1. Combustibles movilizados por la empresa Terpel, que diariamente transporta 455 mil galones para abastecer las diferentes aeronaves (Terpel, s. f.). Se debe tener en cuenta que los dos tipos de combustibles utilizados impactan globalmente el ambiente. En el 2011, las emisiones $\mathrm{de} \mathrm{CO}_{2}$ producidas por la industria de la aviación en el mundo fueron de 676 millones de toneladas (Amezcua, Gutiérrez \& Aburto, 2016). 
Desde los años noventa, la aviación nacional en Colombia ha crecido significativamente debido a la implementación de nuevas aerolíneas comerciales y de bajo coste, lo que significa el aumento del tráfico aéreo e implica un incremento en las emisiones contaminantes. Un informe de Estudios Sectoriales de la Aeronáutica Civil, reportó que en el 2019 Colombia incrementó en un 9,1 \% la movilización de pasajeros por vía aérea en relación al 2018 (Mintransporte, 2020). Adicionalmente, gracias a datos de la Aeronáutica Civil se tiene que en los últimos diez años el país ha duplicado el número de rutas a destinos internacionales y ha mostrado un incremento de $168,4 \%$ en el número de pasajeros movilizados (Portafolio, 2018). A pesar del gran crecimiento del sector aéreo en el país, hoy en día, según la Unidad Administrativa Especial de Aeronáutica Civil (UAEAC), en Colombia no están establecidas políticas que promuevan el uso y la producción de combustibles alternativos para la aviación.

En el 2013 la aerolínea LAN Colombia, hoy en día conocida como Latam Colombia, realizó el primer vuelo nacional de corto alcance en la ruta Bogotá-Cali con duración de 1 hora en una aeronave A320-200, abastecida con una mezcla de $69 \%$ JET-A1 y $31 \%$ biocombustible a base de aceite de camelina, con el fin de poder reducir $\mathrm{CO}_{2}$ y otros componentes químicos que afectan al medio ambiente. El vuelo fue un éxito y la aerolínea está en el proceso de implementar dicha mezcla de biocombustible en sus aeronaves, sin embargo, hasta el 2019 no se registran evidencias del uso de biocombustibles en la flota actual de las aerolíneas que operan en el país (Vélez, 2013).

Durante enero y junio del 2019, la aviación en Colombia creció de forma satisfactoria. En la actualidad, las aerolíneas nacionales que operan en el país tienen una variedad de aeronaves de diferentes fabricantes como Airbus, Boeing, Embraer y ATR. Como se observa en la tabla 1, de las siete aerolíneas, Avianca es la compañía con más aeronaves en el país, aporta un 48,3\% de las operaciones, según el boletín de la Aeronáutica Civil (Aeronáutica Civil de Colombia, 2019). Esta compañía al igual que todas las demás, utiliza combustible JET-A1 en todas sus aeronaves, lo cual corresponde al 99,7 \% del total de consumo energético de la compañía (Avianca Holdings, 2017; 2018).

Tabla 1.

Flota de cada aerolínea nacional de Colombia

\begin{tabular}{|c|c|c|c|c|c|c|c|}
\hline $\begin{array}{l}\text { Aeronaves/ } \\
\text { Aerolíneas }\end{array}$ & Avianca & Latam Colombia & $\begin{array}{c}\text { Copa Airlines } \\
\text { Colombia }\end{array}$ & Satena & Easyfly & $\begin{array}{l}\text { VivaAir } \\
\text { Colombia }\end{array}$ & Wingo \\
\hline A318-100 & 10 & 0 & 0 & 0 & 0 & 0 & 0 \\
\hline A319-100 & 25 & 10 & 0 & 0 & 0 & 0 & 0 \\
\hline A320-200 & 61 & 5 & 0 & 0 & 0 & 0 & 0 \\
\hline A320 NE0 & 10 & 0 & 0 & 0 & 0 & 0 & 0 \\
\hline A321-200 & 13 & 0 & 0 & 0 & 0 & 17 & 0 \\
\hline A321-NEO & 2 & 0 & 0 & 0 & 0 & 0 & 0 \\
\hline A330-200 & 9 & 0 & 0 & 0 & 0 & 0 & 0 \\
\hline B787-900 & 1 & 0 & 0 & 0 & 0 & 0 & 0 \\
\hline ATR 42 & 2 & 0 & 0 & 7 & 4 & 0 & 0 \\
\hline ATR 72 & 15 & 0 & 0 & 0 & 15 & 0 & 0 \\
\hline B737-700 & 0 & 0 & 3 & 0 & 0 & 0 & 4 \\
\hline ERJ 145 & 0 & 0 & 0 & 2 & 0 & 0 & 0 \\
\hline ERL 170 & 0 & 0 & 0 & 1 & 0 & 0 & 0 \\
\hline ERJ 190 & 0 & 0 & 2 & 0 & 0 & 0 & 0 \\
\hline B787-800 & 13 & 0 & 0 & 0 & 0 & 0 & 0 \\
\hline Total & 161 & 15 & 5 & 10 & 19 & 17 & 4 \\
\hline
\end{tabular}

Fuente: Boletín de la Aeronáutica Civil de Colombia (2019). 
En Informe Anual del 2018, Avianca explicó que ha implementado soluciones correctivas para mitigar el impacto de las emisiones de $\mathrm{CO}_{2}$. Dentro de estas soluciones, la compañía desarrolló una restauración de su flota con el fin de ayudar a reducir el impacto ambiental con nuevas aeronaves de tipo A320-251 NEO, las cuales reducen el consumo de combustible en un $15 \%$ en relación a los tradicionales A320. Además, gracias a la incorporación de estas aeronaves, los kilogramos de $\mathrm{CO}_{2}$ por cada 100 pasajeros transportados por kilómetro recorrido disminuyeron un 5,43\% respecto al 2016 y un $1,31 \%$ con respecto al 2017 , como se puede visualizar en la figura 2:

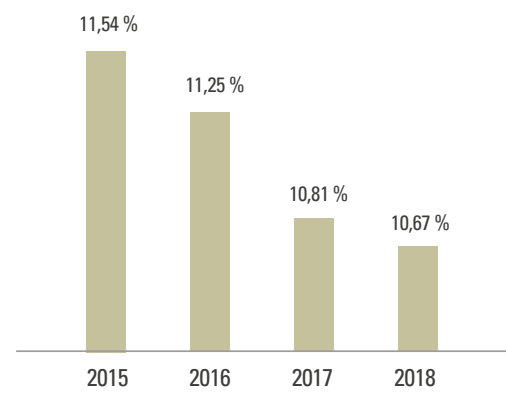

Figura 2. Niveles de $\mathrm{CO}_{2}$ producidos por Avianca en los últimos años Fuente: Avianca Holdings (2018; 2019).

En otro estudio, se evaluó teóricamente la influencia del uso de combustibles alternativos en motores a pistón de aviación. Se tuvieron en cuenta las propiedades químicas de diferentes mezclas entre AVGAS 100/130 con etanol obtenido a partir de caña de azúcar. Del estudio se concluyó que la implementación de combustibles alternativos puede llegar a reducir las emisiones de $\mathrm{CO}_{2}$ hasta un $76 \%$. Sin embargo, se especificó que las mezclas con concentraciones de etanol superiores al $20 \%$ podrían llegar a ser peligrosas para la operación del motor en la fase de crucero, esto debido a un fenómeno conocido como Vapor Lock (Mónico, Vega \& Leyton, 2016).

Por lo anterior, el país debe estar a la vanguardia con respecto a la comunidad internacional, los combustibles alternativos son un componente esencial para afrontar los retos de consumo, contaminación, disminución de ruido, etcétera. De manera análoga, al conocer que Colombia es un país altamente productor de biocombustibles, se han venido implementando acciones y proyectos que permiten hacer uso de combustibles alternativos en la industria aeronáutica, desde el 2008 se idealizó la política de implementación de dichos carburantes, y a la fecha, existen combustibles en estudio de certificación para la operación normal de aeronaves comerciales a pesar de que el país se ha centrado en la producción de biocombustibles para la industria automotriz.

A su vez, para el 2018 se estimaba un gran avance en el uso de biocombustibles derivados de aceites vegetables no comestibles como la jatropha o la camelina (Fedebiocombustibles, 2012b). Sin embargo, según expertos, el uso de este tipo de carburantes no sería rentable para las aerolíneas sino hasta el 2025. No obstante, las compañías aéreas han mostrado interés en el uso de combustibles alternativos como solución a las problemáticas ambientales. Aunque frente a ese aspecto, a la fecha, no existe en Colombia evidencia alguna de que las aerolíneas tengan en uso combustibles alternativos (Fedebiocombustibles, 2012b).

Por último, las diferentes refinerías de combustibles alternativos que existen en el país se deben instruir y certificar en la norma ASTM 7566, con el fin de producir biocombustibles que cumplan con las especificaciones necesarias para soportar la operación aérea y dar cumplimiento a los estándares internacionales (Guzmán-Galeano, 2013).

\section{Conclusiones}

En los últimos 15 años, en el ámbito mundial las empresas líderes en el diseño y producción de aeronaves y motores junto con algunas compañías aéreas, han desarrollado proyectos en pro de buscar nuevas fuentes de energía que sustituyan el combustible fósil. Los resultados obtenidos hasta este momento demuestran que los biocombustibles son una muy buena alternativa a futuro, por ser un combustible más amigable con 
el medio ambiente, sin perjudicar de forma importante las prestaciones de los motores. Lo ideal es que en la industria aeronáutica se potencialice el uso de biocombustibles de segunda generación, es decir, aquellos derivados de plantas no comestibles y que se pueden cultivar en zonas desérticas, para no afectar la producción de alimentos de consumo humano.

En el ámbito internacional, trabajos en conjunto de aerolíneas y fabricantes de motores en los últimos 20 años han realizado importantes avances en ensayos experimentales, que han comprobado que los biocombustibles a futuro, pueden ser un excelente sustituto del combustible de origen fósil. Sin embargo, se deben seguir las investigaciones para hacerlo más competitivo y fácil de emplear en los motores de aviación, sin perjudicar las prestaciones de los motores, ya sea de pistón o a reacción.

A pesar de que Colombia es uno de los grandes productores de biocombustibles en Latinoamérica, competitivos en producto y calidad, a la fecha aún no existe en el país una política que busque o motive a las empresas del sector aéreo a utilizar combustibles alternativos. Cabe resaltar que, a pesar de esto, en los últimos 5 años el país ha empezado a tomar realmente interés en el empleo de biocombustibles, pero no se han tenido avances importantes en este sector y requiere del interés de empresas, entidades y academia, para que en un futuro cercano la industria de la aviación nacional pueda emplear y beneficiarse de las ventajas de emplear este tipo de combustibles más amigables con el medio ambiente.

\section{Referencias bibliográficas}

Aeronáutica Civil de Colombia. (2019). Boletines operacionales. http://www.aerocivil.gov.co/atencion/estadis ticas-de-las-actividades-aeronauticas/boletines-opera cionales

Amezcua, M., Gutiérrez, J., \& Aburto, J. (2016). Combustibles avanzados de aviación. Instituto Mexicano del Pétróleo. http://rtbioenergia.org.mx/wp-content/uploads/ 2016/12/Divulgacion_Biojetfuel-booklet-vf.pdf
Avianca Holdings S.A. (2018, marzo). Informe anual 2017. http:// s22.q4cdn.com/896295308/files/doc_financials/2017/ annual/04/19/Informe-Avianca-Holdings-ESPA\%C3\% 910L-VF.pdf

Avianca Holdings S.A. (2019). Informe anual 2018. http://s22. q4cdn.com/896295308/files/doc_financials/2018/AVH_2018-Ab-4_compressed.pdf

Beta Analytic. (s. f.). Biocombustibles para la aviación: el futuro de los combustibles para aviones. https://www. betalabservices.com/espanol/biocombustibles/com bustibles-para-aviacion.html

Boeing estudia producir en Medio Oriente nuevos biocombustibles con plantas del desierto. (s. f.). Fedebiocombustibles. https://www.fedebiocombustibles.com/notaweb-id-1663.htm

Boeing se asocia con South African Airways para obtener combustible de una nueva planta de tabaco. (s. f.). Fedebiocombustibles. https://www.fedebiocombustibles.com/ nota-web-id-1892.htm

Briceño, D., \& López, O. (2011). Importancia del combustible en el transporte aéreo colombiano. Universidad Militar Nueva Granada. https://repository.unimilitar.edu.co/ bitstream/10654/3217/2/BricenoMorenoDianaCaroli na2011.pdf

China permite uso comercial de biocombustible para aviación. (s. f.). Fedebiocombustibles. https://www.fedebio combustibles.com/nota-web-id-1685.htm

Fedebiocombustibles. (2011). Air France vuela con biocombustibles. https://www.fedebiocombustibles.com/notaweb-id-908.htm

Fedebiocombustibles. (2012a). Biocombustibles para la aviación podrían ser competitivos en menos de una década. https://www.fedebiocombustibles.com/nota-web-id1051.htm

Fedebiocombustibles. (2012b). Biocombustibles para la aviación podrían ser competitivos en menos de una década. https://www.fedebiocombustibles.com/notaweb-id-1026.htm

Fedebiocombustibles. (2013). Firman convenio para el desarrollo de biocombustible aeronáutico [en Argentina]. https://www.fedebiocombustibles.com/nota-webid-1336.htm

Ferrer, R. (2020, 27 de enero). Combustibles aeronáuticos. Aertec Solutions. https://aertecsolutions.com/2020/01/ 27/combustibles-aeronauticos/

Guzmán-Galeano, W. (2013). Bienvenida a las bioenergías en la aviación colombiana [Tesis de especialización]. Universidad Militar Nueva Granada. https://n9.cl/kzgxw 
Martín-Crespo, L. (2016). Biocombustibles en aviación. Hispaviación. http://www.hispaviacion.es/biocombustiblesen-aviacion/

México apuesta por biocombustible en aviación. (s. f. ). Fedebiocombustibles. https://www.fedebiocombustibles. com/nota-web-id-580.htm

Mintransporte. (2020, 21 de febrero). En 9,1\% aumentó el tráfico de pasajeros movilizados vía aérea en 2019. https:// n9.cl/uyu0

Mónico-Muñoz, L., Vega, J., \& Leyton, J. (2016, 22 de julio). Bio-Ethanol Fuel Mixtures Theoretical Influence on Aviation Reciprocating Engines [ponencia]. 14th International Energy Conversion Engineering Conference, AIAA Propulsion and Energy Forum, 1-9. https://doi.org/10. 2514/6.2016-4954

Mónico-Muñoz, L. (2013). Contribución al estudio del ruido de combustión en conceptos avanzados de combustión diesel [Tesis Doctoral no publicada]. Universidad Politécnica de Valencia.

Mónico-Muñoz, L., Cabezas-Paredes, J., \& Buitrago-Triana, S. (2018). Desarrollo de una metodología para calcular los niveles de emisiones contaminantes en motores de combustión interna alternativos. Ciencia y Poder Aéreo, 13(1), 54-71. https://doi.org/10.18667/cienciaypoderaereo.586

Mucino, F. (2017, 20 de diciembre). Así avanzan los biocombustibles en la aviación. A21. https://a21.com.mx/ aeronautica/2017/12/20/asi-avanzan-los-biocombusti bles-en-la-aviacion

Organización de Aviación Civil Internacional [OACI]. (2016, 17 de junio). Tendencias presentes y futuras respecto al ruido y las emisiones de las aeronaves. https://www.icao. int/Meetings/a39/Documents/WP/wp_055_es.pdf
Organización de Aviación Civil Internacional [OACI]. (2017, 18 de septiembre). Conferencia sobre la aviación y los combustibles alternativos. https://www.icao.int/Meetings/ CAAF2/Documents/CAAF.2.WP.020.4.es.pdf

Portafolio. (2016, 5 de diciembre). Energías renovables, la apuesta que debe hacer el país. Portafolio. https://www. portafolio.co/innovacion/energias-renovables-en-co lombia-502061

Portafolio. (2018, 28 de febrero). En 10 años, Colombia duplicó su conexión aérea con el mundo. Portafolio. https:// www.portafolio.co/negocios/en-10-anos-colombia-du plico-su-conexion-aerea-con-el-mundo-514765

Repsol. (s. f. ). Biocombustibles para volar más limpio. https:// www.repsol.com/imagenes/global/es/Biocombusti bles\%20para\%20volar\%20m\%C3\%A1s\%20limpio_ tcm13-15270.pdf

Rodríguez, M. (2016, 28 de enero). Biocombustibles en la aviación. Escuela de Organización Industrial. https://www. eoi.es/blogs/merme/biocombustibles-en-la-aviacion/

Terpel. (s. f. ). Aviación Terpel. https://www.terpel.com/en/ home-Productos-y-Servicios/Aviacion/Aviacion-Terpel/

Torregrosa, A. J., Broatch, A., Plá, B., \& Mónico, L. F. (2013). Impact of Fischer-Tropsch and Biodiesel Fuels on Trade-offs Between Pollutant Emissions and Combustion Noise in Diesel Engines. Biomass and Bioenergy, 52, 22-33. https://doi.org/10.1016/j.biombioe.2013.03.004

Vélez, L. (2013, 21 de agosto). LAN realiza primer vuelo con biocombustible en Colombia. C el Colombiano. https:// www.elcolombiano.com/historico/lan_realiza_primer_ vuelo_con_biocombustible_en_colombia-NCec_25 6568 